\title{
Effects of leaning workstation on oxygenation in the prefrontal cortex and cognitive performance
}

\author{
Veera Aneesh Kuppam', In-Sop Kim²*, Sai Akhil Penumudi', Jaejin Hwang' \\ ${ }^{1}$ Department of Industrial and Systems Engineering, Northern Illinois University, DeKalb, IL; ${ }^{2}$ Allied Health and Communicative Disorders/Northern \\ Illinois University, DeKalb, IL., USA
}

Purpose: The objective of this study was to evaluate the effect of different workstations on prefrontal brain activity and cognitive performance during standardized neurocognitive tasks. Methods: With a repeated-measures laboratory study, 20 university students $(24.5 \pm 3.0$ years old; 10 females) were exposed to either a conventional sitting workstation or leaning workstation. After a one-hour exposure to the workstation, participants performed five different neurocognitive tasks which assessed the executive function and working memory.

Results: The oxygenation levels in the prefrontal cortex were significantly different between the two workstations ( $p$ 's $<0.042$ ). However, there was no significant difference in cognitive performance (speed and accuracy) between the two workstations ( $p$ 's >0.064). Given higher oxygenation concentration in the prefrontal cortex, the leaning workstation might have improved the capacity of executive function and working memory when compared to the sitting workstation.

Conclusions: The findings suggest that leaning workstation may improve the cognitive function when compared to sitting workstation by increasing the prefrontal cortex activity associated with the executive function and working memory. This study exposed the potential neurocognitive benefits of the leaning workstation.

Keywords: Leaning workstation, Active workstation, Cognitive function, fNIR, Brain activity

\section{INTRODUCTION}

Children and adults in the United States typically spend $55 \%$ of their waking time as a sedentary behavior [1]. Prolonged sitting and sedentary work have been associated with many negative health outcomes including the risk of work-related musculoskeletal disorders (MSDs) [2], cardiovascular disorders [3], impaired cognition [4], and type II diabetes [5]. It is known that moderate to vigorous physical activity could lower the rates of morbidity and mortality [6].

To make sedentary work more dynamic, several studies have examined the efficacy of various engineering controls such as the sit-stand desks and walking while working [7-9]. It was found that these interventions could significantly reduce the sitting time and improve the posture and productivity, although the long-term health benefits have not been proven. Previous studies have shown that capillary blood glucose responses and energy expenditure of 10 subjects were significantly alternated during standing deskwork than a sitting deskwork [7]. Even though standing or walking while working

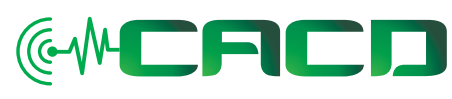

Received: June 26, 2019 Revision: August 9, 20119 Accepted: August 12, 2019

\section{Correspondence:} In-Sop Kim

Allied Health and Communicative Disorders/Northern Illinois University, 1425 Lincoln Hwy, DeKalb, IL, USA Tel: +1-815-753-7793

Fax: +1-815-753-9123

E-mail: ikim@niu.edu

(C) 2019 The Korean Association of SpeechLanguage Pathologists

This is an Open Access article distributed under the terms of the Creative Commons Attribution NonCommercial License (http://creativecommons.org/ licenses/by-nc/4.0/) which permits unrestricted noncommercial use, distribution, and reproduction in any medium, provided the original work is properly cited. 
might be an attractive intervention to combat sedentary behavior, these options might not be practical for many employees due to the potential disturbance to their jobs [10].

The dynamic sitting strategy has recently gained interest as an innovative approach to induce movement for people who seldom get up from their desks [11]. Several manufacturers have designed office furniture that directly encourages an office worker to incorporate fidgeting movements while working at their usual business tasks [12]. Previous studies showed that leaning (perching) workstation induced pelvic movement and reduced spinal loads of 20 subjects when compared to sitting or standing workstations, which showed potential biomechanical advantages of this intervention [8]. The task performed by subjects was limited to a typing task.

Not only the biomechanical benefits but also physical activity intervention could improve the cognitive function [13]. Increased cerebral blood flow due to exercise could improve cognitive functions including executive functions and working memory by changing the brain's activities $[14,15]$. For instance, a school aerobic exercise program increased bilateral prefrontal cortex activity, and improved the executive function and mathematics achievement among overweight 7 to 11 year old children $(N=171)$ [14]. A randomized control trial was employed and dose response was not addressed in this study. A functional magnetic resonance imaging (fMRI) measurement showed that the activities in the prefrontal cortex played a role in executive functioning and working memory $[16,17]$. Thus, it is assumed that increasing the energy expenditure through dynamic sitting might influence executive functions and working memory. However, there has been a lack of evidence showing how an active workstation (leaning workstation) could affect frontal brain activity and cognitive function.

Therefore, the objective of this study was to evaluate the effect of two different office workstations (conventional sitting and leaning workstations) on the frontal brain activity and cognitive performance (executive function and working memory) during standardized neurocognitive tasks. We hypothesized that a leaning workstation would increase the oxygenation in the prefrontal cortex and improve the performance of cognitive tasks in comparison to a sitting workstation.

\section{METHODS}

\section{Participants}

A total of 20 university students (10 males and 10 females) were recruited throughout the community and participated in this study. The mean and standard deviation of age, height, and weight were $24.5 \pm 3.0$ years old, $168.5 \pm 9.8 \mathrm{~cm}$, and $77.4 \pm$ $21.8 \mathrm{~kg}$, respectively. All participants did not have current (past 7 days) musculoskeletal pain or a history of musculoskeletal disorders. The study was approved by Institutional Review Board, and all participants were asked to give their written consent prior to the data collection. After completion of a data collection, compensation for participation was provided.

\section{Experimental protocol}

In a between-subject design, 20 participants with an equal gender distribution were exposed to either the conventional sitting workstation or the leaning workstation to minimize carryover effects of cognitive tasks. For the sitting workstation, the chair, table, and monitor were adjusted to fit each participant based on ANSI/HFES 100-2007 standards [18]. For the leaning workstation (Locus, Focal Upright Furniture, North Kingstown, RI), the pivoting single-post seat and the heightadjustable table (with 5 degrees of inclination) were provided. Per the manufacturer's recommendation, the seat height was adjusted relative to the participant's vertical inseam height to obtain a thigh-to-trunk angle of $135^{\circ}[8,19]$. In order to adopt the assigned workstation (sitting or leaning workstation), participant performed typical computer work tasks for 1-hour: 15-minute typing; 15-minute editing; 15-minute typing; and 15-minute editing using a traditional desktop computer setting (keyboard and mouse with monitor) [19]. When their 1-hour of exposure was complete, an fNIR device was placed on the participant's forehead. A neurocognitive test battery via the psychology experiment building language (PEBL) was conducted for 25 minutes to assess the executive function and working memory [20].

\section{fNIR data}

Prefrontal cortex activity was measured using the fNIR device (fNIR 400 system; Biopac Systems, inc.; Santa Barbara, CA). The fNIR band (4 light sources and 10 photodetectors) was placed on the participants' foreheads (over the eyebrows) based on the international 10-20 EEG procedure of electrode placement F7, Fp1, Fp2, and F8, and Brodmann's areas 9, 10, 45 , and 46 [21]. The fNIR band was secured with a flexible head band. The cognitive optical brain imaging (COBI) studio software was utilized to measure and process the hemodynamic activity of 16 active optodes [22]. For the fNIR data pro- 
cessing, the relative concentrations of the hemodynamic responses $(\mu \mathrm{mol} / \mathrm{L})$ in the prefrontal cortex were evaluated per task using the modified Beer-Lambert law [23]. The raw light intensity measures were filtered using the first low pass filter with a cut off of $0.1 \mathrm{~Hz}$ and motion artifact induced by head or body movement was removed by using a sliding window motion artifact rejection (SMAR) system with fNIRSoft [24].

Prior to each task, baseline cerebral oxygenation values were recorded for each participant to normalize the values. During the baseline session, participants closed their eyes for 30 seconds while seated. The normalized oxygenated hemoglobin $\left(\mathrm{HbO}_{2}\right)$, total hemoglobin $(\mathrm{HbT})$, reduced hemoglobin $(\mathrm{HbR})$, and oxygenation changes (Oxy: difference between $\mathrm{HbO}_{2}$ and $\mathrm{HbR}$ ) of the prefrontal cortex were analyzed. These hemodynamic measures were grouped into left (optodes from 1 to 8 ) and right (optodes from 9 to 16) hemisphere for a regional analysis. For each side of hemisphere, the mean value of the corresponding optodes was calculated.

\section{Cognitive performance}

Five different cognitive tasks including Wisconsin Cart Sort, Flanker, Memory Span, Trail-Making, and Stroop Color Word tasks were used in this study. Participants were asked to perform the task as quickly and accurately as possible. In the Wisconsin Cart Sort task [25], cards were sorted based on three different rules including the number of shapes (from 1 to 4), color of shapes (red, green, blue, and yellow), and the shape (triangle, star, plus, and circle). These rules were unknown and changed during the task. Participants were required to properly sort these multi-attribute cards, and the "correct" or "incorrect" feedback was displayed in the screen each time. In Flanker task [26], participants were instructed to determine the direction of a center arrow among 5 arrows. Flankers were displayed with either congruent (arrows are pointing in the same direction) or incongruent (arrows are pointing in the opposite direction) conditions. In the Memory Span task [27], up to 9 different named images (apple, bus, deer, pear, tree, bird, chipmunk, fish, and plane) were displayed in a sequence. After seeing all images (minimum number of images was 3), participants were required to click the images in the correct order. If the answer was correct, the additional image was displayed in the next trial (maximum number of images was 9). In Trail-Making task [28], participants were asked to connect dots in three different ways including numbers (from 1 to 26), letters (from A to Z), and alternating number-letter. In Stroop Color Word task [25], par- ticipants were instructed to determine the color of the stimulus, which had 4 different colors (red, green, blue, and yellow) and color names (matched or not matched with the stimulus color).

As an output measure, the mean reaction time (milliseconds) of Wisconsin Cart Sort (correct and incorrect), Flanker (congruent and incongruent), and Stroop Color Word tasks was measured. The proportion of correct response (\%) was measured of all five tasks. The total completion time (seconds) of Trail-Making test (number, letter, and number-letter) was analyzed.

\section{Data analysis}

Normality of the fNIR data and cognitive performance measures was initially diagnosed by Shapiro-Wilks W tests in SPSS (version 24; IBM Corporation, Armonk, NY). If the data had non-normality, logarithm or Johnson transformation was applied. The two-way repeated measures Analysis of Variance (ANOVA) was conducted on $\mathrm{HbO}_{2}, \mathrm{HbT}, \mathrm{HbR}$, and Oxy. The workstation and hemisphere were determined as fixed effects and the participant was set as a random effect. The paired $t$ test was used to evaluate the effect of workstation (sitting vs. leaning) on cognitive performance. Due to the non-normality even after transformation, Wilcoxon signed rank tests were used for the correct responses (\%) in Flanker and Trail-Making tasks. The statistical significance was determined with $p$ value $<0.05$.

\section{RESULTS}

\section{fNIR data}

In Memory Span task, the leaning workstation showed significantly higher $\mathrm{HbO}_{2}$ and $\mathrm{HbT}$ compared to the sitting condition ( $p$ 's $<0.039$ ), as seen in Table 1 and Figure 1. In Trail-Making task, $\mathrm{HbO}_{2}$ and Oxy were significantly higher with the leaning workstation than the sitting condition ( $p$ 's $<0.042$ ). Figure 2 shows the Oxy for each optode among participants during Trial-Making task between the sitting and leaning workstations. Other tasks did not show differences in fNIR measures according to workstation. In Trail-Making task, the left hemisphere showed significantly higher $\mathrm{HbT}$ and $\mathrm{HbR}$ compared to the right hemisphere ( $p$ 's $<0.033$ ). In general, there were no significant workstation $\times$ hemisphere interactions with the exception of the Oxy during Wisconsin Card Sort task $(p=0.041)$. There were greater Oxy in the right hemisphere compared to the left hemisphere while using the lean- 
Table 1. $p$-values from the ANOVA analyses of $\mathrm{HbO}_{2}, \mathrm{HbT}, \mathrm{HbR}$, and $\mathrm{Oxy}$ by the workstation, hemisphere, and workstation $\times$ hemisphere two-way interaction

\begin{tabular}{|c|c|c|c|c|}
\hline $\begin{array}{l}\text { Cognitive } \\
\text { Task }\end{array}$ & Measures & Workstation & Hemisphere & $\begin{array}{c}\text { Workstation } \times \\
\text { Hemisphere }\end{array}$ \\
\hline \multirow[t]{4}{*}{ WCS } & $\mathrm{HbO}_{2}$ & 0.479 & 0.735 & 0.055 \\
\hline & $\mathrm{HbT}$ & 0.962 & 0.864 & 0.679 \\
\hline & $\mathrm{HbR}$ & 0.292 & 0.737 & 0.224 \\
\hline & Oxy & 0.271 & 0.664 & $0.041^{*}$ \\
\hline \multirow[t]{4}{*}{$\mathrm{F}$} & $\mathrm{HbO}_{2}$ & 0.777 & 0.377 & 0.730 \\
\hline & $\mathrm{HbT}$ & 0.497 & 0.642 & 0.494 \\
\hline & $\mathrm{HbR}$ & 0.759 & 0.802 & 0.561 \\
\hline & $0 x y$ & 0.999 & 0.444 & 0.866 \\
\hline \multirow[t]{4}{*}{ MS } & $\mathrm{HbO}_{2}$ & $0.039^{*}$ & 0.825 & 0.594 \\
\hline & $\mathrm{HbT}$ & $0.036^{*}$ & 0.508 & 0.590 \\
\hline & $\mathrm{HbR}$ & 0.948 & 0.324 & 0.646 \\
\hline & $0 x y$ & 0.056 & 0.391 & 0.879 \\
\hline \multirow[t]{4}{*}{ TM } & $\mathrm{HbO}_{2}$ & $0.042^{*}$ & 0.974 & 0.596 \\
\hline & $\mathrm{HbT}$ & 0.070 & $0.033^{*}$ & 0.688 \\
\hline & $\mathrm{HbR}$ & 0.824 & $0.014^{*}$ & 0.944 \\
\hline & Oxy & $0.010^{*}$ & 0.120 & 0.707 \\
\hline \multirow[t]{4}{*}{ SCW } & $\mathrm{HbO}_{2}$ & 0.289 & 0.469 & 0.199 \\
\hline & $\mathrm{HbT}$ & 0.486 & 0.461 & 0.757 \\
\hline & $\mathrm{HbR}$ & 0.225 & 0.520 & 0.087 \\
\hline & Oxy & 0.312 & 0.693 & 0.134 \\
\hline
\end{tabular}

Significance level $\left(^{*}\right)$ was set at $p<0.05$.

WCS, Wisconsin Cart Sort; F, Flanker; MS, Memory Span; TM, Trail-Making; SCW, Stroop Color Word. ing workstation, which was opposite to the trend of the sitting condition (Figure 3).

\section{Cognitive performance}

There were no significant changes of cognitive performance measures (mean reaction time, correct response, and total time) between sitting and leaning workstations ( $p$ 's >0.064), as shown in Table 2.

\section{DISCUSSION AND CONCLUSIONS}

This study evaluated the influence of two different workstations (sitting vs. leaning) on hemodynamic response in the prefrontal cortex and cognitive performance during standardized neurocognitive assessment tasks. The results showed that the leaning workstation significantly increased oxygenation levels during Memory Span (working memory) and Trail-Making (language and numerical processing) tasks as compared with the sitting workstation. There was no significant difference in cognitive performance between sitting and leaning workstations.

Greater $\mathrm{HbO}_{2}$ (oxygenated hemoglobin) and $\mathrm{HbT}$ (total hemoglobin) were observed in the leaning workstation compared to the sitting workstation during Memory Span task. Memory Span task is designed to assess the working memory, short-term memory, and visual processing [27]. The leaning workstation required a greater activation on the prefrontal cortex (especially in the central right region: $p$-value of optode
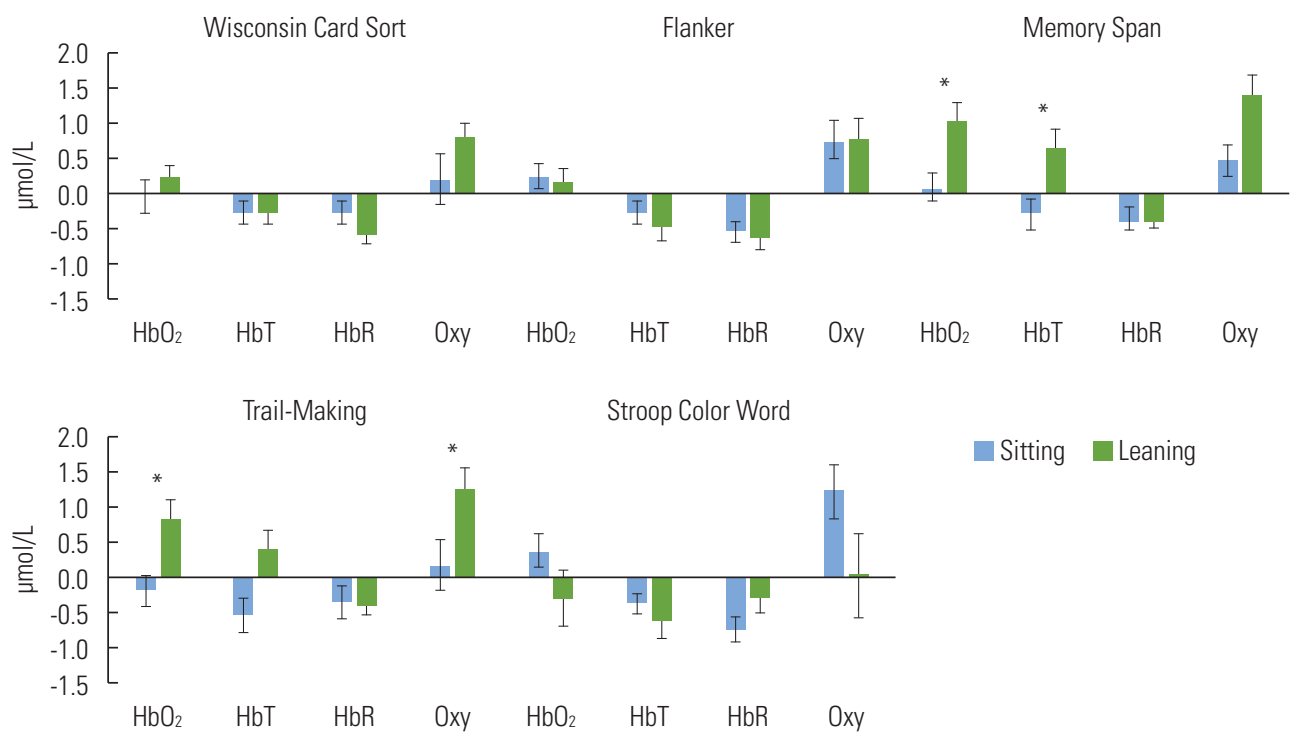

Figure 1. Mean and standard error of $\mathrm{HbO}_{2}, \mathrm{HbT}, \mathrm{HbR}$, and $\mathrm{Oxy}$ of five different cognitive tasks (W
and Stroop Color Word) by workstation (sitting vs. leaning). Significance level $\left({ }^{*}\right)$ was set at $p<0.05$. 

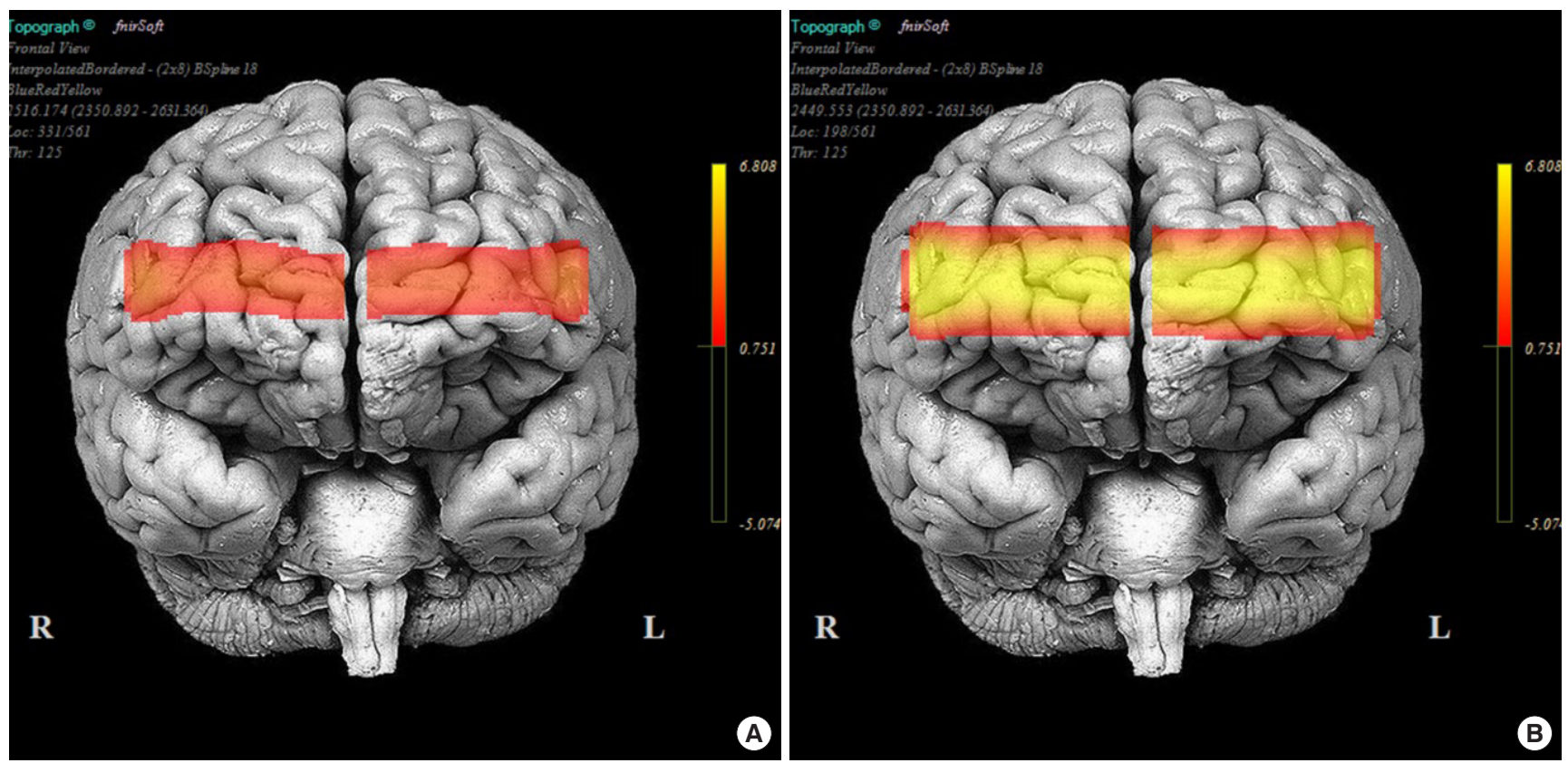

Figure 2. Topographic representation of Oxy values in Trail-Making task over brain surface image. (A) Sitting workstation; (B) leaning workstation. The yellow color indicates higher Oxy values in the prefrontal cortex area.

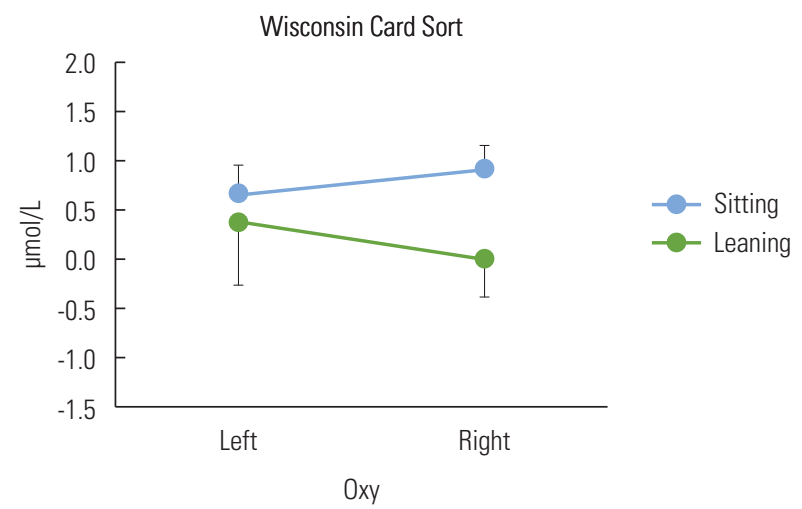

Figure 3. Interaction plot of Oxy during Wisconsin Card Sort task by workstation and hemisphere. Error bars represent the standard error.

12 of $\mathrm{HbT}=0.022)$ than the sitting workstation during a working memory task. This was similar to previous studies showing that stand-biased desk exposure (28 weeks period) increased brain activation $\left(\mathrm{HbO}_{2}\right)$ among high school students during Memory Span task [29]. In addition, activation in the right-hemisphere prefrontal area was known to be associated with spatial working memory processes [30]. This suggests that the leaning workstation may increase the working memory capacity by increasing the prefrontal activity on the right hemisphere.

During Trail-Making task, the leaning workstation showed greater $\mathrm{HbO}_{2}$ and Oxy (oxygenation changes) on the prefron-
Table 2. Mean (standard error) of cognitive performance measures (mean reaction time, correct response, and total time) by workstation (sitting vs. leaning). $p$-values from the paired $t$-tests were summarized.

\begin{tabular}{lccc}
\hline Measures & Sitting & Leaning & $p$ \\
\hline Mean reaction time (ms) & & & \\
WCS Correct & $1,111.4(68.1)$ & $1,027.9(49.0)$ & 0.431 \\
WCS Incorrect & $1,295.0(103.0)$ & $1,196.3(75.1)$ & 0.564 \\
FCongruent & $407.6(13.0)$ & $404.4(17.3)$ & 0.853 \\
F Incongruent & $454.1(12.9)$ & $456.6(18.3)$ & 0.905 \\
SCW & $873.5(48.8)$ & $829.4(81.7)$ & 0.704 \\
Correct response (\%) & & & \\
WCS & $84.5(1.2)$ & $85.9(1.0)$ & 0.282 \\
FCongruent & $95.7(1.8)$ & $98.9(0.7)$ & 0.098 \\
F Incongruent & $91.4(2.0)$ & $93.6(1.5)$ & 0.404 \\
SCW & $98.0(0.7)$ & $95.8(0.6)$ & 0.064 \\
MS & $54.3(2.8)$ & $53.3 .(2.3)$ & 0.789 \\
TM Number & $98.2(0.5)$ & $98.5(0.4)$ & 0.887 \\
TM Letter & $98.7(0.5)$ & $97.8(0.7)$ & 0.272 \\
TM Number-Letter & $96.8(0.8)$ & $95.7(1.1)$ & 0.341 \\
Total Time (seconds) & & & \\
TM Number & $83.1(4.4)$ & $76.9(1.7)$ & 0.316 \\
TM Letter & $80.1(3.5)$ & $80.5(2.5)$ & 0.530 \\
TM Number-Letter & $104.2(7.0)$ & $105.7(5.8)$ & 0.869 \\
\hline
\end{tabular}

WCS, Wisconsin Cart Sort; F, Flanker; MS, Memory Span; TM, Trail-Making; SCW, Stroop Color Word. 
tal cortex (especially in the central left region: $p$-value of optode 8 of $\mathrm{HbO}_{2}=0.016$ ) compared to the sitting workstation. The Trail-Making task is designed to assess the language and numerical processing, and hand-eye coordination [31]. Previous study showed that stand-biased desk exposure increased the $\mathrm{HbO}_{2}$ in the left hemisphere during the Trail-Making task compared to the conventional sitting desk, which is similar to our findings [29]. It was found that a lower $\mathrm{HbO}_{2}$ was associated with the slower reaction in the executive condition [32]. This indicates that the leaning workstation may improve the executive function in left frontal cortex compared to the sitting workstation by increasing the oxygenation levels in the leftprefrontal brain.

Physical activity interventions are known to be associated with the improvements in working memory, executive function, and increased prefrontal cortex activation [14,29,33]. Although we did not measure the movement of participants while using the leaning workstation, previous studies have shown that a leaning workstation significantly increased the postural transitions of the pelvis (lateral and anterior/posterior directions) compared to the sitting workstation [8]. It indicates that a leaning workstation induced pelvic movements of participants, which could be associated with increased brain activation in the prefrontal cortex.

The cognitive performance of five different tasks were not significantly different between the sitting and leaning workstations, which was not consistent with the results of the fNIR data. This indicates that changes of hemodynamic activity in the prefrontal cortex may not necessarily relate to the behavior outcomes of cognitive tasks, which was similar to the previous study's finding during Wisconsin Cart Sort task [34]. Moreover, we only recruited healthy young university students. Given relatively lower task complexity for university students, this task might not effectively distinguish the cognitive performance between two workstations. Previous studies found a significant difference of cognitive performance (executive function and working memory) among freshmen high school students after using stand-biased desks [29]. This might suggest that more complex cognitive load tasks might be suitable to evaluate the cognitive function among healthy young adults.

Although this study was carefully designed and conducted, some limitations are recognized. First, a small sample size (10 participants for each workstation) was included in this study, which could affect the statistical significance of our measures. Future study could consider using a larger sample size to gen- eralize our findings. Second, participants experienced the short exposure duration (two hours) to each workstation. Studying the long-term effects of a leaning workstation on the neurocognitive function could be merited in future study.

In conclusion, the leaning workstation increased the oxygenation levels in the prefrontal cortex compared to the conventional sitting workstation during neurocognitive tasks assessing the executive function and working memory. Enhanced pelvic movements in the leaning workstation may improve the capacity of the executive function and working memory by increasing cerebral blood flow in the prefrontal cortex. Future study could investigate the long-term impacts of leaning workstation on neurocognitive function.

\section{CONFLICT OF INTEREST}

None.

\section{REFERENCES}

1. Matthews CE, Chen KY, Freedson PS, Buchowski MS, Beech BM, Pate RR, et al. Amount of time spent in sedentary behaviors in the United States, 2003-2004. Am J Epidemiol 2008;167:875-881.

2. Andersen JH, Fallentin N, Thomsen JF, Mikkelsen S. Risk factors for neck and upper extremity disorders among computer users and the effect of interventions: an overview of systematic reviews. PLoS One 2011;6:e19691.

3. Katzmarzyk PT, Church TS, Craig CL, Bouchard C. Sitting time and mortality from all causes, cardiovascular disease, and cancer. Med Sci Sports Exerc 2009;41:998-1005.

4. Falck RS, Davis JC, Liu-Ambrose T. What is the association between sedentary behaviour and cognitive function? A systematic review. Br J Sports Med 2016:bjsports-2015.

5. Hamilton MT, Hamilton DG, Zderic TW. Role of low energy expenditure and sitting in obesity, metabolic syndrome, type 2 diabetes, and cardiovascular disease. Metab Syndr Relat Disord 2008;6:81-82.

6. Kesaniemi YA, Danforth E, Jensen MD, Kopelman PG, LefÈbvre P, Reeder BA. Dose-response issues concerning physical activity and health: an evidence-based symposium. Med Sci Sports Exerc 2001;33:S351-S358.

7. Buckley JP, Mellor DD, Morris M, Joseph F. Standing-based office work shows encouraging signs of attenuating post-prandial glycaemic excursion. Occup Env Med 2013:oemed-2013-101823. doi:10.1136/oemed-2013-101823.

8. Le P, Marras WS. Evaluating the low back biomechanics of three different office workstations: Seated, standing, and perching. Appl Ergon 2016;56:170-178.

9. Noma H, Ohmura A, Kuwahara N, Kogure K. Wearable sensors for auto-event-recording on medical nursing-user study of ergo- 
nomic design. Wearable Comput. 2004 ISWC 2004 Eighth Int. Symp. On, vol. 1, IEEE; 2004, p. 8-15.

10. Júdice PB, Hamilton MT, Sardinha LB, Silva AM. Randomized controlled pilot of an intervention to reduce and break-up overweight/obese adults' overall sitting-time. Trials 2015;16:490.

11. Mörl F, Bradl I. Lumbar posture and muscular activity while sitting during office work. J Electromyogr Kinesiol 2013;23:362-368.

12. Pynt J. Rethinking design parameters in the search for optimal dynamic seating. J Bodyw Mov Ther 2015;19:291-303.

13. Donnelly JE, Lambourne K. Classroom-based physical activity, cognition, and academic achievement. Prev Med 2011;52:S36S42.

14. Davis CL, Tomporowski PD, McDowell JE, Austin BP, Miller PH, Yanasak NE, et al. Exercise improves executive function and achievement and alters brain activation in overweight children: a randomized, controlled trial. Health Psychol 2011;30:91.

15. Villringer A, Dirnagl U. Coupling of brain activity and cerebral blood flow: basis of functional neuroimaging. Cerebrovasc Brain Metab Rev 1995;7:240-276.

16. Carpenter PA, Just MA, Reichle ED. Working memory and executive function: Evidence from neuroimaging. Curr Opin Neurobiol 2000;10:195-199.

17. Cohen JD, Perlstein WM, Braver TS, Nystrom LE, Noll DC, Jonides J, et al. Temporal dynamics of brain activation during a working memory task. Nature 1997;386:604.

18. ANSI/HFES 100. ANSI/HFES 100-2007. (2007). Human factors engineering of computer workstations. 2007.

19. Kingston DC, Riddell MF, McKinnon CD, Gallagher KM, Callaghan JP. Influence of input hardware and work surface angle on upper limb posture in a hybrid computer workstation. Hum Factors 2016;58:107-119.

20. Mueller ST, Piper BJ. The psychology experiment building language (PEBL) and PEBL test battery. J Neurosci Methods 2014;222:250-259.

21. Rodrigo AH, Ayaz H, Ruocco AC. Examining the neural correlates of incidental facial emotion encoding within the prefrontal cortex using functional near-infrared spectroscopy. Int. Conf. Augment. Cogn., Springer; 2016, p. 102-112.

22. Ayaz H, Shewokis PA, Bunce SC, Onaral B. Functional near infrared spectroscopy based brain computer interface, 2018.
23. Cope M, Delpy DT. System for long-term measurement of cerebral blood and tissue oxygenation on newborn infants by near infra-red transillumination. Med Biol Eng Comput 1988;26:289-294.

24. Izzetoglu M, Devaraj A, Bunce S, Onaral B. Motion artifact cancellation in NIR spectroscopy using Wiener filtering. IEEE Trans Biomed Eng 2005;52:934-8. doi:10.1109/TBME.2005.845243.

25. Huizinga M, Dolan CV, van der Molen MW. Age-related change in executive function: Developmental trends and a latent variable analysis. Neuropsychologia 2006;44:2017-2036.

26. vel Grajewska BŻ, Sim EJ, Hoenig K, Herrnberger B, Kiefer M. Mechanisms underlying flexible adaptation of cognitive control: Behavioral and neuroimaging evidence in a flanker task. Brain Res 2011;1421:52-65.

27. Dempster FN. Memory span: Sources of individual and developmental differences. Psychol Bull 1981;89:63.

28. Shibuya-Tayoshi S, Sumitani S, Kikuchi K, Tanaka T, Tayoshi S, Ueno SI, et al. Activation of the prefrontal cortex during the TrailMaking Test detected with multichannel near-infrared spectroscopy. Psychiatry Clin Neurosci 2007;61:616-621.

29. Mehta RK, Shortz AE, Benden ME. Standing up for learning: A pilot investigation on the neurocognitive benefits of stand-biased school desks. Int J Environ Res Public Health 2015;13:59.

30. Jonides J, Smith EE, Koeppe RA, Awh E, Minoshima S, Mintun MA. Spatial working memory in humans as revealed by PET. Nature 1993;363:623-625.

31. Reitan RM. Trail Making Test: Manual for administration and scoring. Reitan Neuropsychology Laboratory; 1992.

32. Mekari S, Fraser S, Bosquet L, Bonnéry C, Labelle V, Pouliot P, et al. The relationship between exercise intensity, cerebral oxygenation and cognitive performance in young adults. Eur J Appl Physiol 2015;115:2189-2197.

33. Kamijo K, Pontifex MB, O'Leary KC, Scudder MR, Wu CT, Castelli DM, et al. The effects of an afterschool physical activity program on working memory in preadolescent children. Dev Sci 2011;14: 1046-1058.

34. Cinciute S, Daktariunas A, Ruksenas O. Hemodynamic effects of sex and handedness on the Wisconsin Card Sorting Test: the contradiction between neuroimaging and behavioural results. PeerJ 2018;6:e5890. 